\title{
Save Energy in Wireless Sensor Networks Routing Using Fuzzy Approach and Biogeography Based Optimization
}

\author{
Yasser Kareem Al-Rikabi \\ Assistant lecturer, College of Computer Science and IT, University of Sumer, Iraq
}

yasseralhilali2@gmail.com

\begin{abstract}
Extending the lifetime of the energy constrained wireless sensor networks is a crucial challenge in wireless sensor networks (WSNs) research. When designing a WSN infrastructure Resource limitations have to be taken into account. The inherent problem in WSNs is unbalanced energy consumption, characterized by multi hop routing and a many-to-one traffic pattern. This uneven energy dissipation can significantly reduce network lifetime. This paper proposes a new routing method for WSNs to extend network lifetime using a combination of a fuzzy approach and Biogeography Based Optimization (BBO) algorithm which is capable of finding the optimal routing path form the source to the destination by favoring some of routing criteria and balancing among them to prolong the network lifetime. To demonstrate the effectiveness of the proposed method in terms of balancing energy consumption and maximization of network lifetime, we compare our approach with the BBO search algorithm and fuzzy approach using the same routing criteria. Simulation results demonstrate that the network lifetime achieved by the proposed method could be increased by nearly $25 \%$ more than that obtained by the BBO algorithm and by nearly $20 \%$ more than that obtained by the fuzzy approach.
\end{abstract}

\section{Keywords}

Biogeography Based Optimization (BBO) algorithm, fuzzy approach, network lifetime, routing, wireless sensor networks (WSNs).

\section{INTRODUCTION}

Awireless sensor network is a network of distributed independent plans that can sense or monitor physical or environmental settings cooperatively [1]. Large scale in numerous applications used on wireless sensor networks such as environmental monitoring, remote habitat monitoring, detection of natural calamities and her prediction, structural health monitoring and medical monitoring [2]. Sensor nodes in the wide-scale data-gathering networks are usually power-driven by small and inexpensive batteries in anticipation of living for a long period [3]. Other military sensor networks include acoustic sensor arrays for antisubmarine warfare such as the fixed distributed system and the advanced deployable system, and unattended ground sensors such as the remote battlefield sensor system and the deployment-are tackled when low-cost sensors and communication networks become available [4]. A wireless sensor network consists of certain amount of small and energy-constrained nodes. Basic components of a sensor node include a single or multiple sensor modules, a wireless transmitter-receiver module, a computational module, and a power supply module. Such networks are normally deployed for data collection where human intervention after deployment, to recharge or replace node batteries, may not be feasible, resulting in limited network lifetime. Fig. 1 shows the plan diagram of components inside a characteristic sensor node that comprises of sensing, processing, transmission, mobilizes, location finding system and power units. It also shows the communication construction of a WSN. Each sensor node makes its decisions based on its mission, the information it currently has, knowledge of its computing, communication, and energy resources. The node must have ability to collect and route data either to other nodes or back to an external base station or stations that may be a fixed or a mobile node capable of connecting the sensor network to an existing communication substructure or to the internet [5].

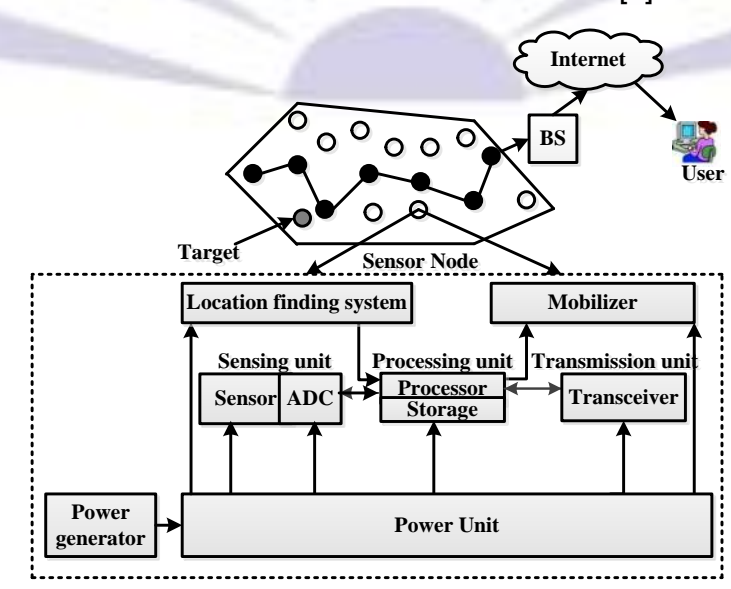

Fig. 1: Components of a sensor node 
L ternational Journal of Management and Information Technology In traditional optimal path routing schemes over WSNs, each node selects fixed nodes to relay data according to some criteria in order to maximizing network lifetime. Therefore, a good routing method in WSNs involves finding the optimal transmission path form the sender through relay nodes to the destination in order to prolong the network lifetime. Due to this conception, the lifetime problem in WSNs has received significant attention in the recent past. Due to limitations in the communication range, sensors communicate with each other (possibly) through multi-hop wireless communication links and forward sensed data on behalf of others so the sink can receive them on time for further processing and analysis. Since, each sensor node acts as a routing element for other nodes for transmitting data.

The challenge in designing of a sensor network is that, sensors must be organized into a robust multi-hop wireless network that should be able to function properly for a long period of time. This, in general, is a difficult problem since sensors have limited computing capabilities and very limited power supply. Uneven Energy Consumption (UEC) is an inherent problem in WSNs characterized by the multi-hop routing and many-to-one traffic pattern. The network lifetime can significantly reduce by UEC [6-7].

For WSNs routing protocol design, the energy efficiency and energy balance should be two different technical goals, since they will lead to routing algorithms with different attributes. An energy-efficient routing protocol tries to prolong the network lifetime through minimizing the energy exhaustion; while an energy-balanced routing protocol intends to extend the network lifetime through uniform energy exhaustion. Previous results easily in the premature network division that disables the network functioning, through there may be much remaining energy left. On the other side, the latter may not be optimal with respect to energy efficiency, as it can burn energy evenly to keep network connectivity and maintain network functioning as long as possible [3], [7-8].

The problem with many routing algorithms is that they minimize the total energy consumption in the network at the expense of non-uniform energy drainage in the networks. Such approaches cause network division because some nodes that are part of the efficient path are drained from their battery energy quicker. In many cases, the lifetime of a sensor network is over as soon as the battery power in critical nodes is depleted [6]. Therefore, in this paper, the proposed method seeks to investigate the problems of balancing energy consumption and maximization of network lifetime for WSNs. We propose a new approach by combining Fuzzy approach and Biogeography Based Optimization algorithm by selected the optimal routing path from the source to the destination by favoring highest remaining energy and the lowest distance (minimum number of hops) to the sink. This paper is organized as follows. In Section 2 present the Related work and Related concepts of the routing algorithm to maximum the WSN lifetime. This paper describes a brief background of Fuzzy approach and Biogeography Based Optimization algorithm in section 3. Section 4 presents the routing model for the proposed routing method. Performance evaluation is proposed in Section 5. Finally, conclusion and discussion are presented in Section 6.

\section{RELATED WORK}

A critical aspect in the design of the heterogeneous WSNs and applying the good routing paths are to save energy and extend the lifetime of the networks [9]. A good routing protocol in WSNs involves finding the optimal transmission path form the sender through relay nodes to the destination in order to prolong the network lifetime. Due to these challenges, the lifetime problem in WSNs has received significant attention in the recent times. Network lifetime of fixed deployment schemes were studied in [10], observed that in wireless sensor networks, the behavior of a single node lifetime demonstrates the nature of normal Gaussian distribution, which brings the first light of exploring the network lifetime behavior given the knowledge of nodes' lifetimes.

The work in [11], addressed the issue of lifetime analysis and estimation for wireless sensor networks in which the sensor nodes are deployed at desired locations. Instead of trying out various probability basis, to apply an interval type-2 fuzzy approach system (FLS) for lifetime analysis and estimation in a wireless sensor network is proposed. The authors [12] proposed a hybrid routing method for a heterogeneous WSN to prolong the lifetime of the network. The hybrid routing combines clustering and multi-hop communication methods, which are nodes placed near to sink perform a multi-hop network and nodes far away from sink perform clusters. In [13], the authors used an ant colony optimization approach to maximize the lifetime of heterogeneous WSNs. The methodology is based on finding the maximum number of disjoint connected covers that satisfy both sensing coverage and network connectivity. The approaches in [14-15] took a different view for prolonging the network-lifetime. They attempt to sustain the availability of the sensors that have less energy by distributing the traffic load to the ones with much residual energy. All of the above-mentioned works focus on improving energy-efficiency using fixed routing paths; nonetheless, due to the lack of path diversity, those nodes traversed by fixed routing paths may drain out their energy quickly. Hence, for selecting cluster [16], the cluster head is not easy in different environments which may have different characteristics (different fuzzy descriptors) including the remaining battery power, number of neighbor nodes, distance from cluster centroid, and network traffics, and evaluated their performance. The sensor nodes closer to the base station consume much more energy due to the increased network traffic near the base station. Hence, the sensor nodes closer to the base station quickly run out of battery. The option for fuzzy approach is justified by the possibility of establishing levels of abstraction to increase the network lifetime with a small penalty to the end-to-end throughput, which can be easily modifiable [17]. More importantly, many low complex fuzzy approach implementations are available in practice [18], which makes the baseband processing suitable for sensor nodes. For instance, some low-complex examples of fuzzy approach based algorithms for WSNs are given in [6], [19], where the authors propose energy efficient clustering and routing schemes. Nevertheless, both works operate into higher network layers, and such clustering and routing 
International Journal of Management and Information Technology algorithms can be used along with relay selection schemes, as complimentary techniques, aiming at energy efficiency in the physical, multiple access, and network layers.

In this work, a new approach (Biogeography-Based Optimization (BBO) algorithm) for dynamic deployment problem for WSNs is proposed. We considered WSNs which combine mobile and static sensors. It is known that the BBO algorithm and its variants work well for many optimization problems [20-23]. The BBO algorithm was initially used on dynamic deployment for WSN. Considering the good performance of the algorithm, use of the BBO algorithm seemed an appropriate approach to enable the sensors in the network to obtain a good coverage in two dimensional space with static and mobile nodes.

In most applications of WSNs, sensor nodes are densely deployed in large areas. Once deployed, nodes can never be recharged or replaced. After depleting their energy, nodes turn to die and stop working. Since networks cannot accomplish assigned missions after nodes die. The lifetime of WSNs is a crucial parameter when evaluating performance of routing protocols [5], [8]. Fig. 2 shows the network partition (one part of the network may become disconnected from the destination) due to the death of some sensor nodes. The maximization of lifetime can be formulated as an optimization problem. The variables of this optimization problem are routing parameters at nodes. When having sensed or asked to relay a data packet, each node needs to transmit this packet to a sink. However, it cannot send the packet directly to sinks except that it is a sink's neighbor. So normally a node needs to choose a neighboring sensor as its next hop. When nodes are chosen as the next hops they will influence the energy consumption of the network as well as the lifetime.

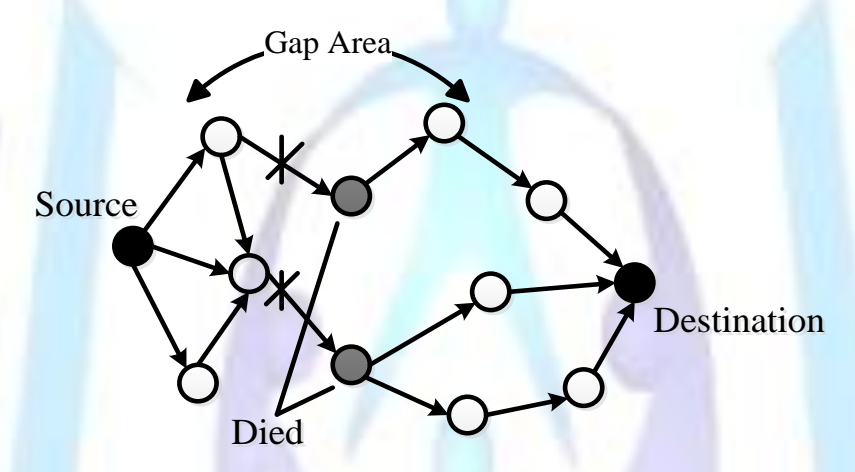

Fig. 2: Network partition due to the death of certain nodes.

In this paper, we also proposed a new routing method using a combination of fuzzy approach and BBO algorithm (Fuzzy_BBO). This method seeks to investigate the problem of balancing energy consumption and maximization of the network lifetime.

\section{FUZZY APPROACH AND BIOLOGEOGRAPHY BASED OPTIMIZATION ALGORITHM 3.1 Fuzzy Approach}

The concept of fuzzy approach was introduced by Zadeh in the mid-1960s [24] as an extension of the concept of an ordinary fuzzy set. Since then, its applications have rapidly expanded in adaptive control systems and system identification. It has the advantages of easy implementation, robustness, and ability to approximate to any nonlinear mapping [25]. In fuzzy systems, the dynamic behavior of a system is characterized by a set of linguistic fuzzy rules based on the knowledge of a human expert, each of which is represented by a linguistic term such as "small," "medium," or "large." Fuzzy sets allow an object to be a partial member of a set. In Fig. 3, if $X$ suggests a collection of objects denoted by $X$, usually $X$ is referred to as the "universe of discourse," and then a fuzzy set $A$ in $X$ is defined by a set of ordered pairs[6]:

$$
A=\left\{\left(\frac{x, \mu_{A}(x)}{x}\right) x \in X\right\}
$$

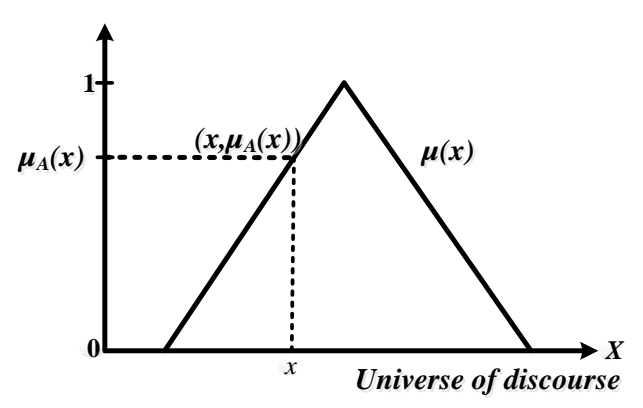

Fig. 3: Membership function from the pair $(x, \mu A(x))$. 
International Journal of Management and Information Technology Where the function $\mu_{A}(x)$ is called membership function of the object $x$ in $A$. This membership function represents a "degree of belongingness" for each object to a fuzzy set, and provides a mapping of objects to a continuous membership value in the interval [0...1]. When a membership value is close to the value $1\left(\mu_{A}(x) \rightarrow 1\right)$ it means that input $x$ belongs to the set $A$ with a high degree, while small membership values $\left(\mu_{A}(x) \rightarrow 0\right)$, indicate that set $A$ does not suit input $x$ very well. In fuzzy systems, the dynamic behavior of a system is characterized by a set of linguistic fuzzy rules based on the knowledge of a human expert.

These rules are the heart of a fuzzy system and may be provided by experts or can be extracted from numerical data. In either case, the rules that we are interested in can be expressed as a collection of IF-THEN statements (IF antecedents THEN consequents). Antecedents and consequents of a fuzzy rule form the fuzzy input space and fuzzy output space respectively are defined by combinations of fuzzy sets. Considering a fuzzy system with $p$ inputs and one output with $M$ rules, then the $L^{\text {th }}$ rule has the form [26]: $R^{L}: I F x_{1}$ is $F_{1}^{L}$ and $\ldots x_{p}$ is $F_{p}^{L}$ THEN $y$ is $G^{L}$ where $F_{1}^{L}$ $\ldots F_{p}^{L}$ and $G^{L}$ denote the linguistic variables defined by fuzzy sets and $L=1 \ldots \mathrm{M}$.

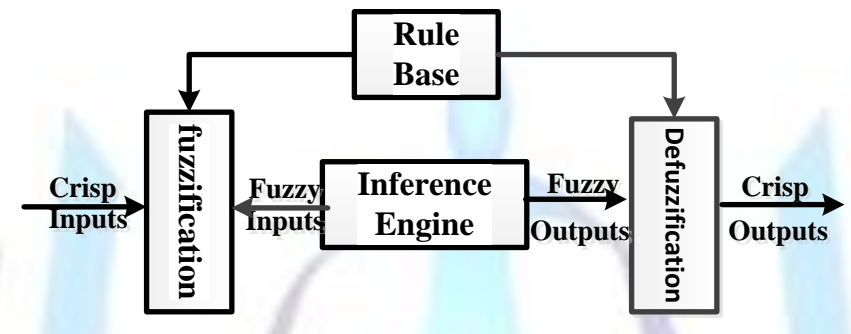

Fig. 4: Typical structure of the fuzzy approach.

Fig. 4 shows the typical structure of a fuzzy system. It consists of four components namely; fuzzification, rule base, inference engine and defuzzification [24]. The processes of making crisp inputs are mapped to their fuzzy representation in the process called fuzzification. This involves application of membership functions such as triangular, trapezoidal, Gaussian etc. The inference engine process maps fuzzified inputs to the rule base to produce a fuzzy output. A consequent of the rule and its membership to the output sets are determined here. The defuzzification process converts the output of a fuzzy rule into crisp outputs by one of defuzzification strategies [6].

\subsection{Biogeography-Based Optimization Algorithm}

The Biogeography-Based Optimization (BBO) algorithm developed by Simon in 2008[27]. The basic premise of this theory is that the rate of change in the number of species on an island critically depends on the balance between the immigration of new species onto the island and the emigration of established species. The BBO algorithm operates in a population of individuals called habitats (or islands). Each habitat represents a possible solution to the problem in hand. The fitness of each habitat is determined by its habitat suitability index (HSI), which is a metric that determines the goodness of a candidate node solution, and each habitat feature is called a suitability index variable (SIV). Good solutions may have a larger number of species, which represents a habitat with a low HSI, than do poor solutions. The immigration rate $\lambda$ and the emigration rate $\mu$ of each habitat are used to probabilistically share information between habitats. These parameters are affected by the number of species $S$ in a habitat. Maximum immigration rate $I$ occur when the habitat is empty and decreases as more species are added and maximum emigration rate $E$ occurs when all possible species Smax are present in the habitat. The immigration and emigration rates when there are $S$ species in the habitat are shown in Fig 5.

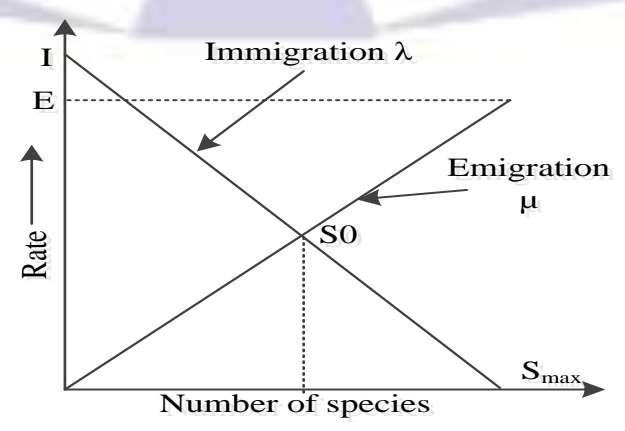

Fig5: Illustration of candidate nodes solutions to some problem 
International Journal of Management and Information Technology In fig 5. The candidate nodes solutions represent as $S_{1}$ represent a low $H S I$ solution, while $S_{2}$ represent a high $H S I$ solution. So, in same figure $S_{1}$ represent a habitat with only a few species, while $S_{2}$ represents a habitat with many species. The immigration rate $\lambda_{1}$ for $S_{1}$ will, therefore, be higher than the immigration rate $\lambda_{2}$ for $S_{2}$. The emigration rate $\mu_{1}$ for $S_{1}$ will be lower than the emigration rate $\mu_{2}$ for $S_{2}$. In BBO, $\lambda_{i}$ is the probability that a given independent variable in the $i$-th candidate solution will be replaced; that is, $\lambda_{i}$ is the immigration probability of $x_{i}$. If an independent variable is to be replaced, then the emigrating candidate solution is chosen with a probability that is proportional to the emigration probability $\mu_{i}$. This probability value is usually performed using roulette wheel selection [27].

$$
P\left(x_{i}\right) \text { is selected for emigration }=\frac{\mu_{i}}{\sum_{n=1}^{N} \mu_{n}}
$$

For $i=1, \ldots N$, where $N$ is the number of candidate solution in the population.

\section{POPOSED ROUTING METHOD}

In this paper, the topology of a WSN is modeled as a directed graph $G(N, A)$, where $N$ is the set of nodes, and $A$ is the set of direct links between the nodes. A sink node is responsible for collecting data from all other nodes within its transmission range [14]. The routing schedule is computed by the base station. It calculates optimal routing schedule and broadcasts it. Every node follows this schedule. The process of finding the optimal path, and broadcasting it in the network and sending data from all nodes to the base station by following this routing schedule is repeated in every round. Computation of routing schedule is done dynamically with the consideration of current level of some criteria of each node. For this, normally it may require the nodes to report their criteria periodically to the base station. The base station can then determine the routing schedule based on this updated information. The proposed method assumes that:

- All sensor nodes are randomly distributed in the area and every sensor node is assumed to know its own position as well as that of its neighbors and the sink.

- All sensor nodes have the same maximum transmission range and the same amount of initial energy.

One of the important measures of WSN is the network lifetime. For the proposed model, whenever any sensor node runs out of energy, communication links between various sensor nodes and the base station will break. This is considered as the end of the network lifetime. Since the lifetime of each sensor node depends on energy consumption, it is important to preserve residual energy of these nodes in such a way that overall network lifetime is extended. The primary goal of this paper is to design a protocol that will prolong the lifetime of the WSNs through limiting energy cost as well as equal distribution of energy consumption. To achieve this, we make use of both the Fuzzy approach and BBO algorithm. This method uses the process consists of two parts.

\subsection{Implementation Of Fuzzy Approach}

In this Section, we focus on describing the major goal of fuzzy approach in proposed protocol. Fuzzy approach is used to calculate the value of the fitness function fit(s) of node $s$ that depends on the remaining energy $R E(s)$ and the distance $D(s)$ to the Sink as shown in Fig.6. Fig.7 shows the proposed method uses five membership functions for

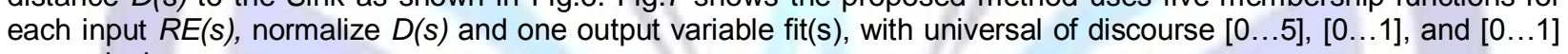
respectively.

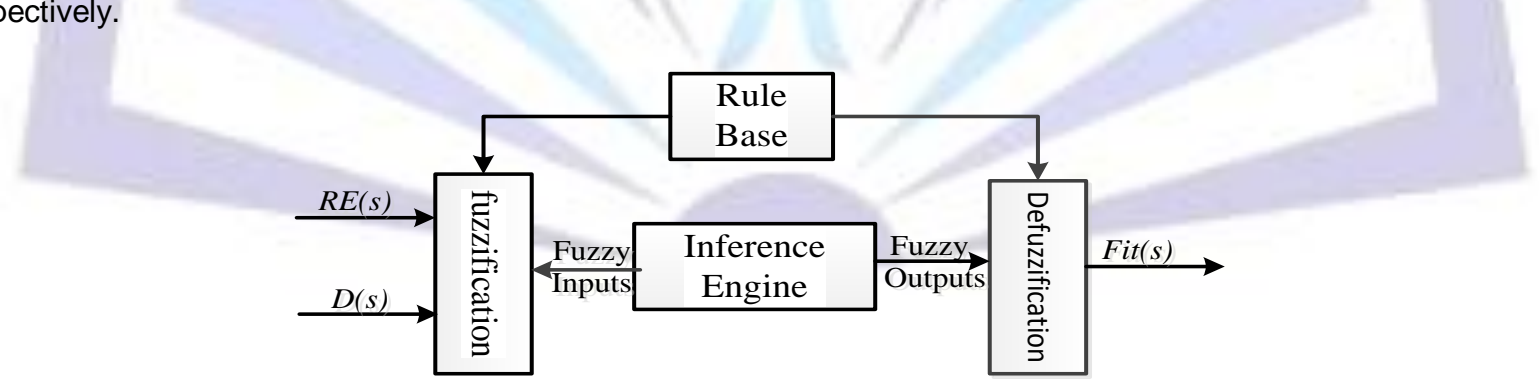

Fig. 6: Fuzzy structure with two inputs (remaining energy and normalize distance to the sink) and one output of the fitness value of nodes.

For the fuzzy approach, the fuzzified values are processed by the inference engine, which consists of a rule base and various methods to inference the rules. Table 1 shows the IF-THEN rules used in the proposed method, with a total number of $5^{2}=25$ for the fuzzy rule base. As an example, IF $R E(s)$ is Very High and $D(s)$ is Very Near THEN Fit(s) is Very Good. All these rules are processed in a parallel manner by a fuzzy inference engine. The defuzzification finds a single crisp output value from the solution fuzzy space. This value represents the fitness function value of node $s$. Practice defuzzification is done using centre-of-gravity method [28] given by: 


$$
f i t(s)=\left(\sum_{k=1}^{n} U_{k} * c_{k}\right) / \sum_{k=1}^{n} U_{k}
$$

Where $U_{i}$ is the output of rule base $k$, and $c_{k}$ is the center of the output membership function.

Table 1. IF-THEN Rules

\begin{tabular}{|c|l|l|l|l|l|}
\hline $\begin{array}{c}\text { RE(s) } \\
\text { D(s) }\end{array}$ & V. Low & Low & Medium & High & V. High \\
\hline \hline V. Near & Normal & Good & Good & V. Good & V. Good \\
\hline Near & Bad & Normal & Good & V. Good & V. Good \\
\hline Medium & V. Bad & Bad & Normal & Good & Good \\
\hline Far & V. Bad & V. Bad & Bad & Normal & Normal \\
\hline V. Far & V. Bad & V. Bad & Bad & Bad & Bad \\
\hline
\end{tabular}
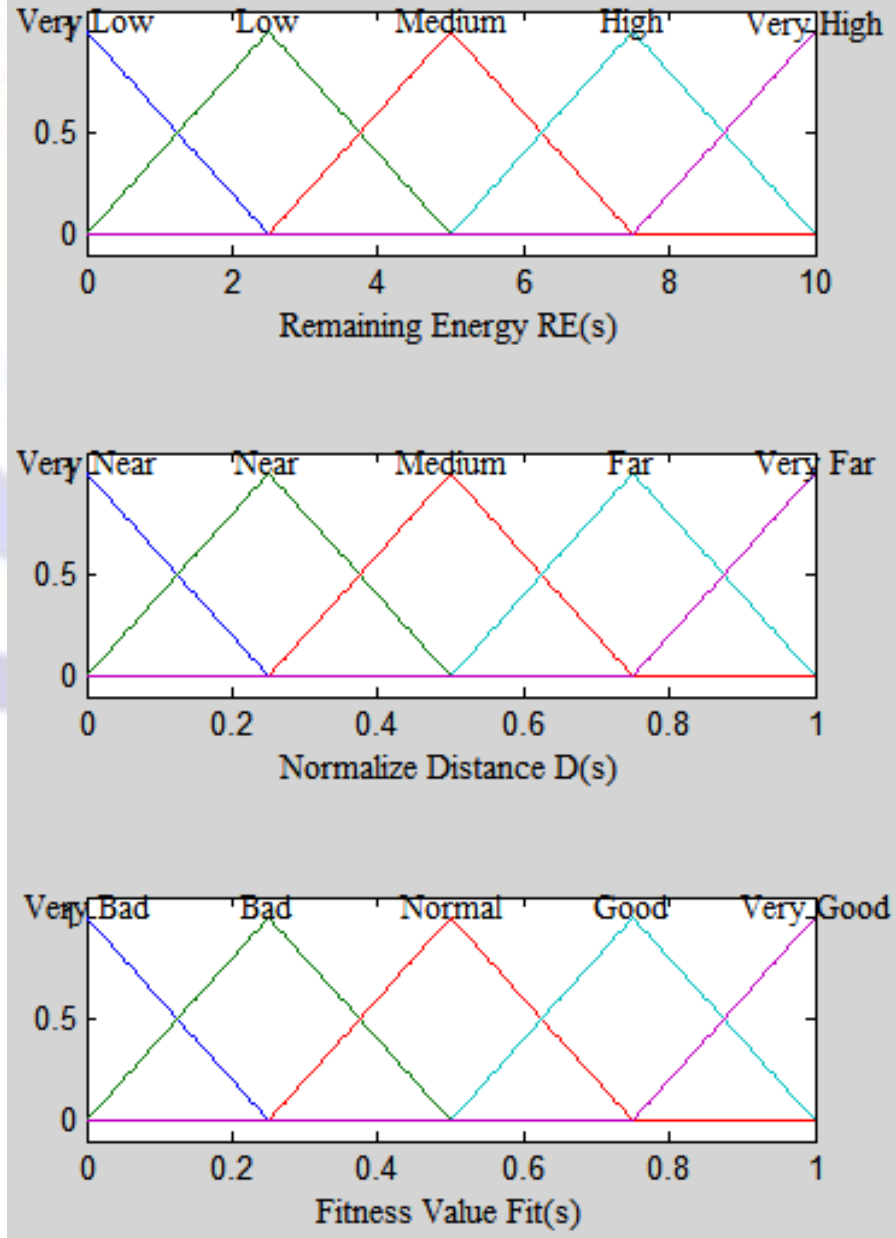

Fig.7: Membership graph for the inputs (remaining energy and normalize distance to the sink) and the output of fitness value of nodes. 


\subsection{Implementation of BBO Algorithm}

Unbalanced energy consumption is an inherent problem in WSNs characterized by the multi-hop routing and many-toone traffic pattern. This uneven energy dissipation can significantly reduce network lifetime. Generally, in routing algorithm, the best path is chosen for transmission of data from source to destination. Over a period, if the same path is chosen for all communications to achieve battery performance in terms of quick transmission time, then those nodes on this path will drain fast. In this paper, we therefore exploit the BBO algorithm to find the optimal routing path for WSNs. This algorithm seeks to investigate the problems of balancing energy consumption and maximization of network lifetime. BBO seeks to find the optimal routing path from the source basic node to the sink. It selects the best node from candidate nodes (neighbors) in the forwarding paths in favoring the highest remaining energy for each node and the short distance (minimum number of hops) to sink. BBO algorithm considers a tree structure in terms of $(S, F)$, where $S$ is the set of candidate solution nodes in the forwarding path and $F$ is the fitness function, which assigns a fitness value $f(s)$ to each candidate solution node $s \in S$.

The fitness value $f(s)$ is determined depending on the remaining energy of node $s$ and the distance from node $s$ to the sink. The tree node is explored on the basis of its fitness value. Where the candidate solution node that has the highest remaining energy and the lowest distance to the sink will be selected as the best node in the forwarding path to the sink. The fitness function $f(s)$ we used is given as follows:

$$
f(s)=\alpha+R E(s)+\frac{1}{\beta \times D(s)}
$$

Where $R E(s)$ is the remaining energy for node $s, D(s)$ is the distance from node $s$ (minimum number of hops) to the destination, and $\alpha, \beta$ are constant values $(\alpha=0.1$ and $\beta=0.16)$. The candidate nodes in BBO algorithm are selected as the next hops (neighbors) of the source where each employed bee is nominated to one of these nodes because each employed bee represents to a candidate node in the following path. All employed bees compute the fitness functions of their nodes and share this information with onlooker bees. According to the equation (2), the onlooker evaluates the information taken from all employed bees and chooses a best node with highest probability $P$ related to its probability value given by the following [27]:

$$
P\left(s_{i}\right)=\frac{f i t\left(s_{j}\right)}{\sum_{j=1}^{N} f i t\left(s_{j}\right)}
$$

Where $P\left(s_{i}\right)$ is the probability value for node $s_{i}, f i t\left(s_{i}\right)$ is the fitness value of the node $s_{i}$, and $N$ is the number of candidate solution nodes equal to the bees number in both employed bees and onlooker bees. The flow chart of the proposed method BBO - Fuzzy is shown in Fig.8.

\section{PERFORMANCE EVALUATION}

To demonstrate the effectiveness of the proposed method (Fuzzy_BBO) in terms of balancing energy consumption and maximizing network lifetime, simulation results of the proposed are compared with those of BBO algorithm and Fuzzy approach. All the approaches use the same routing criteria namely to select the optimal path from the source node to the sink.

The BBO algorithm has shown outperform existing maximum lifetime routing protocols in literatures such as LEACH [29] and Particle Swarm Optimization (PSO) [30]. The fuzzy approach is also shown to exhibit better performance over existing maximum lifetime routing algorithms in literatures such as Online Maximum Lifetime heuristic (OML) [31] and Minimum Transmit Energy (MTE) [32]. Experimental results obtained under various network scenarios in [29-32] indicate that both of BBO algorithm and fuzzy approach give optimal performance in terms of the network lifetime as well as the average energy consumption. 


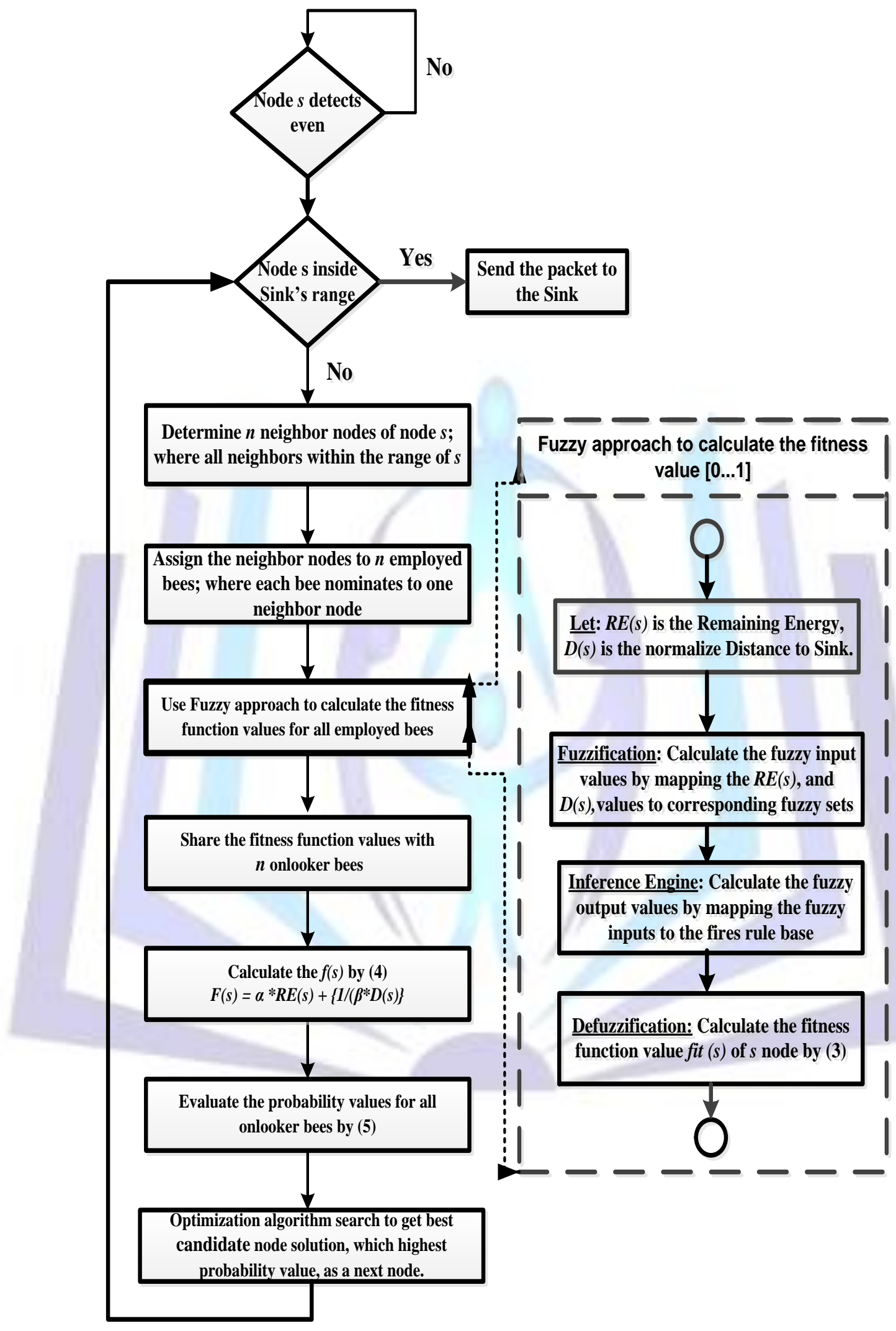

Fig. 8: Flow chart of the proposed algorithm. 


\subsection{Simulation Setup}

The simulations are carried out in MATLAB. 100 sensor nodes are randomly deployed in a topographical area of dimension $100 \mathrm{~m} \times 100 \mathrm{~m}$. this topographical area has the sensed transmission limit of $30 \mathrm{~m}$. The performance of the proposed method is tested in this topographical area. This area is only one data sink which located at $(90 \mathrm{~m}, 90 \mathrm{~m})$. All sensor nodes have the same initial energy $0.5 \mathrm{~J}$. The proposed method uses the first order radio model that is largely used in the area of routing protocol evaluation in WSNs [29]. The transmission and receiving costs are characterized by the expressions.

$$
E_{n} T(k)=E_{\text {elec }} * k+E_{\text {amp }} * k \cdot d^{2} \text { and } E_{n} R(k)=E_{\text {elec }} * \mathrm{k}
$$

respectively, where $k$ is the number of bit per packet, $d$ is the distance from the sender node to the receiver node, $E_{e l e c}$ and $E_{a m p}$ are per bit energy dissipation in transmitting or receiving circuitry and energy required per bit per meter square for the amplifier to achieve acceptable signal to noise ratio (SNR) respectively. Simulations are done using the values $50 \mathrm{~nJ} / \mathrm{bit}$ and $100 \mathrm{pJ} / \mathrm{bit} / \mathrm{m}^{2}$ for $E_{\text {elec }}$ and $E_{a m p}$, respectively. Fig. 9. and Table 2 present the systems parameters in details.

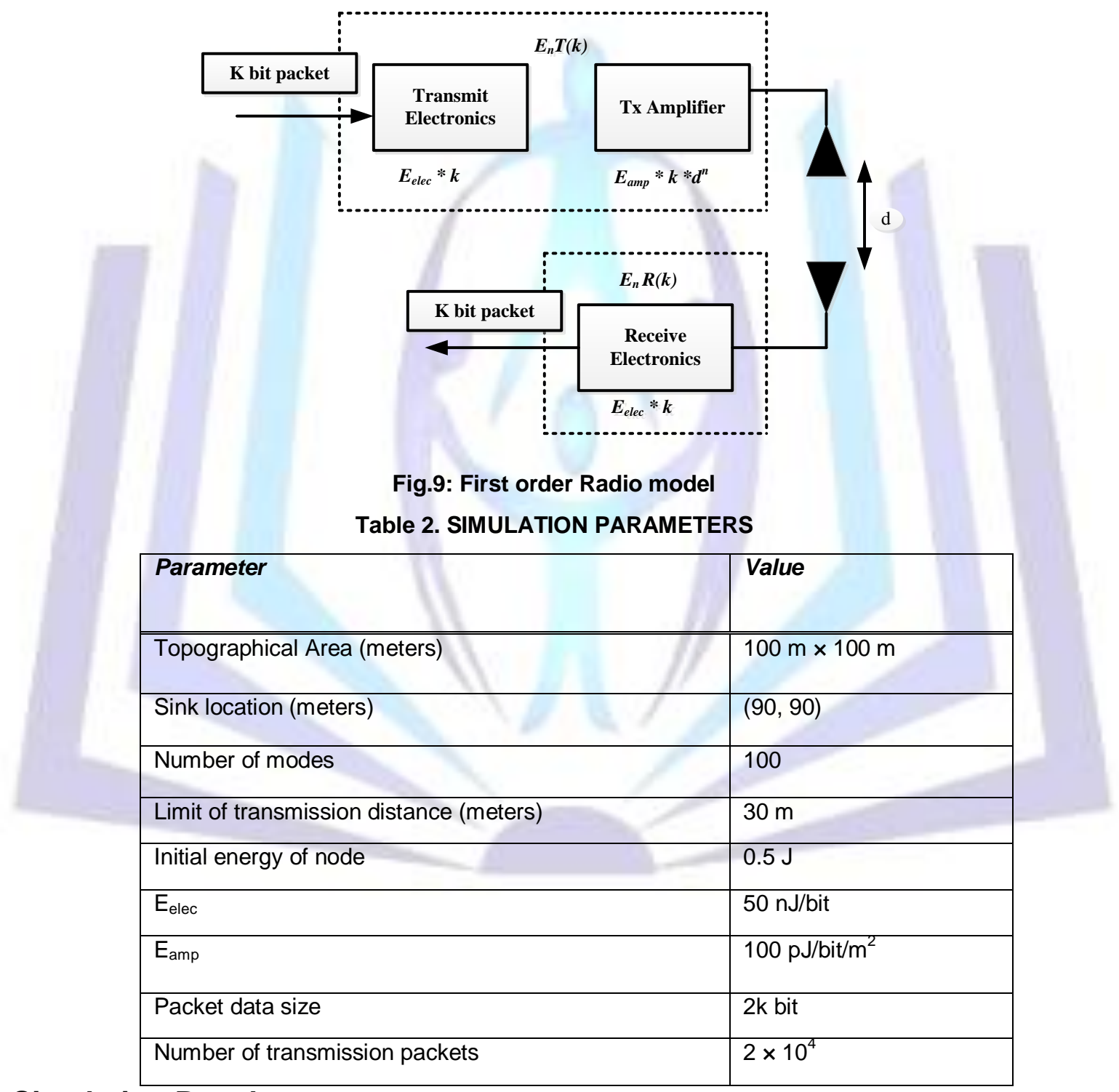

\subsection{Simulation Results}

The number of live nodes as a function of rounds by using the three different approaches for this area is shown in fig. 9. It can be seen that the proposed method outperforms both BBO algorithm and Fuzzy approach in the area. When all packets are sent in area, the network lifetime achieved by the proposed method increased by nearly $29 \%$ than that obtained by BBO algorithm and also increased by nearly $25 \%$ than that obtained by Fuzzy approach. Moreover in fig. 10, it can be seen that the number of alive nodes of the proposed method is always higher than that of both BBO algorithm and Fuzzy approach. 
International Journal of Management and Information Technology The different duration of time corresponding to the first dead node computed using the three different approaches in area is listed in Table 3. Clearly, the time for the first node to die in the proposed method is much longer than the times for the first node to die in both BBO algorithm and Fuzzy approach From fig. 9 and Table 3, it is clear that, the proposed method outperforms both BBO algorithm and Fuzzy approach in terms of balancing energy consumption and maximization of network lifetime.

Table3. NUMBER OF ROUNDS WITH THE FIRST DEAD NODE

\begin{tabular}{|l|l|l|l|}
\hline Approaches & BBO & Fuzzy & $\begin{array}{l}\text { Proposed } \\
\text { method }\end{array}$ \\
\hline $\begin{array}{c}\text { Lifetime of the first } \\
\text { dead node (Rounds) }\end{array}$ & 1384 & 2373 & 6781 \\
\hline
\end{tabular}

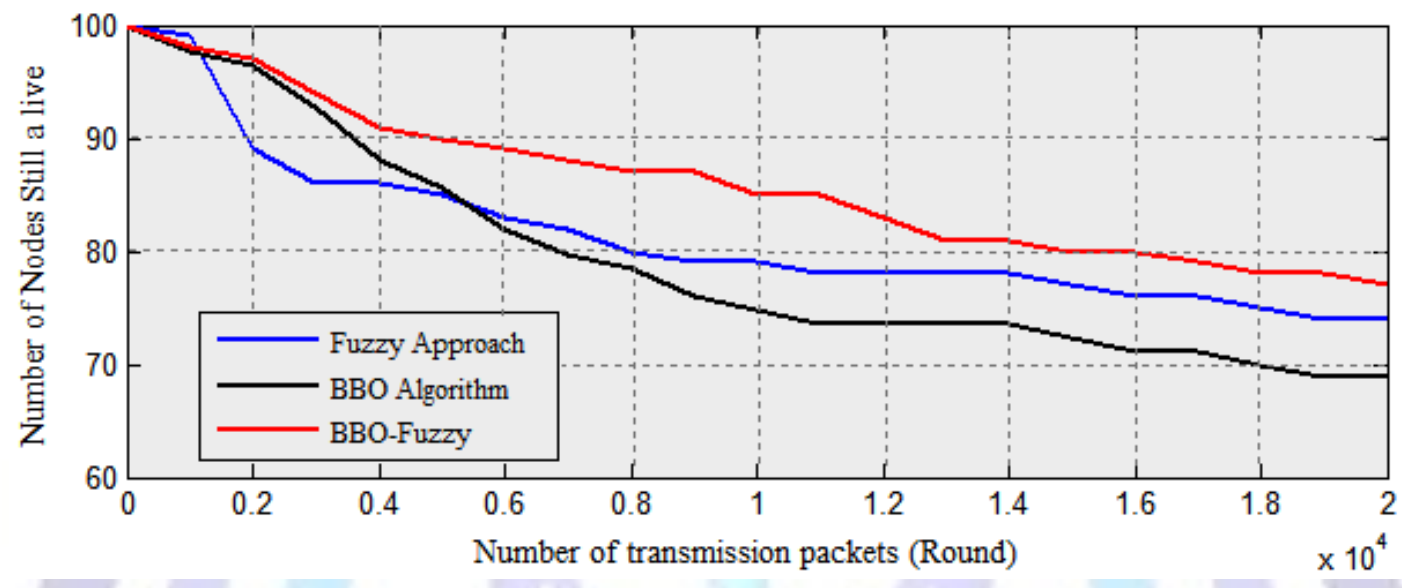

Fig. 10: Number of alive nodes as a function of rounds based on different approaches (BBO, fuzzy, and proposed).

Fig. 11 shows the average remaining energy of a WSN as a function of transmission rounds for the three approaches in area. The proposed method performs better than both A-star algorithm and fuzzy approach [6]. This indicates that, better energy balance in a WSN is achieved by the proposed method in this area The delay incurred in transmission of data packets is also a key parameter for certain applications. The comparison between three different approaches is shown in fig. 12. It can be seen that, the proposed method has shortest delay compared to both BBO algorithm and fuzzy approach. Shorter delay indicates both energy saving and efficient information transmission (especially secure and important ones). In other words, data packets are routed through different node-disjoint paths with multipath routing to avoid network congestion and prolong the network lifetime.

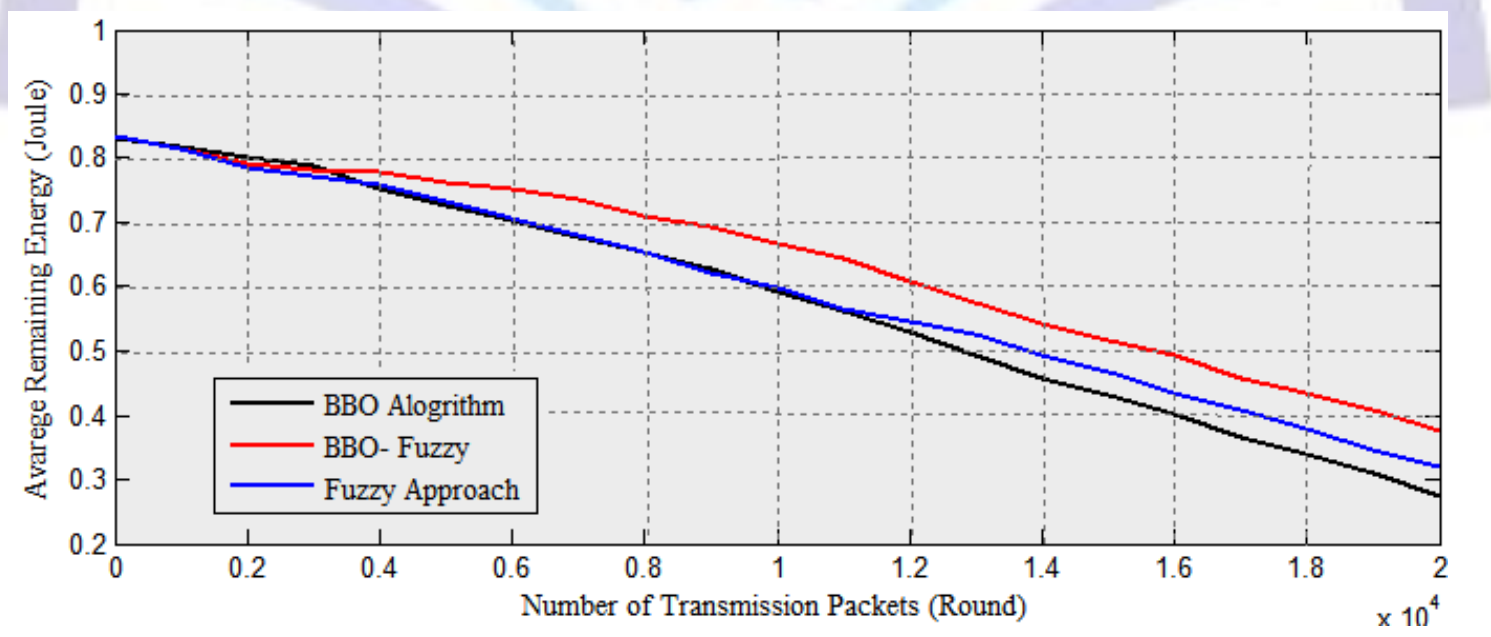

Fig. 11: Average network remaining energy as a function of transmission round. 


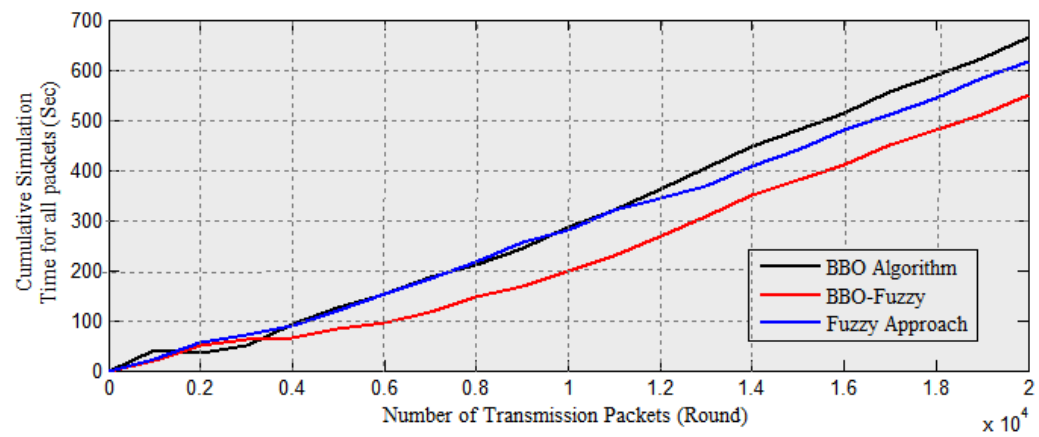

Fig. 12: Data transmission delay (cumulative simulation time for all packets)

\section{as a function of transmission round.}

Note that above simulations are performed assuming that all the nodes are well maintained (i.e. stable with enough power) until the node dies. In real world, there may be certain situations that one even more of the sensors in the critical pathway become intermittent in the ability to function normally. Such behavior may add performance noise (fluctuations) into the WSN. As there are too many parameters to be considered, future investigations about such topics may be quite interesting and challenging.

\section{CONCLUSION}

Resource limitations have to be taken into account when designing a WSN infrastructure. Energy is one of the most critical resources for WSNs. Most of works in the literatures about WSN routing have emphasized energy savings as an important optimization goal. However, where nodes operate in WSNs on limited battery energy efficient utilization of the energy is very important. The lifetime network is highly related to the route selection, this is one of the main characteristics of these networks. Unbalanced energy consumption is an inherent problem in a WSN. To efficiently route data through transmission path from node to node and to prolong the overall lifetime of the network, we proposed a new algorithm by using a combination of both Fuzzy approach and BBO algorithm. The new method is capable of selecting optimal routing path from the source node to the sink by favoring highest remaining energy and minimum number of hops. The performance of this method is evaluated and compared with other two methods under the same criteria. Simulation results demonstrate the effectiveness of the new approach with regards to enhancement of the lifetime of wireless sensor networks with randomly scattered nodes.

\section{REFERENCES}

[1] I. F. Akyildiz, S. Weilian, S. Yogesh, and C. Erdal. "A survey on sensor networks", Communications magazine, IEEE, Vol. 40, No. 8,pp. 102-114, 2002.

[2] C. Y. Chong, and P. K. Srikanta, "Sensor networks: evolution, opportunities, and challenges", Proceedings of the IEEE, Vol. 91, No. 8, pp. 1247-1256, 2003.

[3] H. Zhang, and S. Hong, "Balancing energy consumption to maximize network lifetime in data-gathering sensor networks", Parallel and Distributed Systems, IEEE Transactions, Vol. 20, No. 10, pp. 1526-1539, 2009.

[4] V. A. Kottapalli, S. K. Anne, P. L. Jerome, E. D. Carryer, W. K. Thomas, H. L. Kincho, and L. Ying, "Twotiered wireless sensor network architecture for structural health monitoring." In Smart Structures and Materials, pp. 8-19, 2003..

[5] J. N. Al-Karaki, and E. K. Ahmed, "Routing techniques in wireless sensor networks: a survey", Wireless communications, IEEE, Vol. 11, No. 6, pp. 6-28, 2004.

[6] I. S. AIShawi, Y. Lianshan, P.Wei, and L. Bin, "Lifetime enhancement in wireless sensor networks using fuzzy approach and A-star algorithm”, Sensors Journal, IEEE, Vol. 12, No. 10, pp. 3010-3018, 2012.

[7] F. Ren, Z. Jiao, H. Tao, L. Chuang, and K. R. Sajal, "EBRP: energy-balanced routing protocol for data gathering in wireless sensor networks", Parallel and Distributed Systems, IEEE Transactions, Vol. 22, No. 12,pp. 2108-2125, 2011.

[8] M. Patil, and C. B. Rajashekhar, "A survey on routing protocols in wireless sensor networks", In Networks (ICON)",18th IEEE International Conference, pp. 86-91, 2012.

[9] A. Jamshidi, and N. K. Masoumeh, "Performance analysis of transmitter-side cooperation-receiver-siderelaying schemes for heterogeneous sensor networks", Vehicular Technology, IEEE Transactions, Vol. 57, No. 3, pp. 1548-1563 2008.

[10] E. Jain, and L. Qilian, "Sensor placement and lifetime of wireless sensor networks: theory and performance analysis", In Global Telecommunications Conference, IEEE, vol. 1, pp. 5-pp, 2005. 
nternational Journal of Management and Information Technology

[11] H. Shu, L. Qilian, and G. Jean, "Wireless sensor network lifetime analysis using interval type-2 fuzzy approach systems, Fuzzy Systems", IEEE Transactions, Vol. 16, No. 2, pp. 416-427, 2008.

[12] S. Priyankara, K. Kazuhiko, T. Hideki, and M. Koso, "A clustering/multi-hop hybrid routing method for wireless sensor networks with heterogeneous node types", In GLOBECOM Workshops (GC Wkshps), IEEE, pp. 207-212, 2010.

[13] Y. Lin, Z. Jun, S. H. C. Henry, H. Ip, Wai, L. Yun, and H. S. Yu, "An ant colony optimization approach for maximizing the lifetime of heterogeneous wireless sensor networks," Systems, Man, and Cybernetics, Part C: Applications and Reviews, IEEE Transactions, Vol.42, No. 3,pp. 408-420,2012.

[14] J. Park, and S. Sartaj, "An online heuristic for maximum lifetime routing in wireless sensor networks", Computers, IEEE Transactions, Vol. 55, No. 8, pp.1048-1056, 2006.

[15] C. Wu, Y. Ruixi, and Z. Hongchao, "A novel load balanced and lifetime maximization routing protocol in wireless sensor networks", In Vehicular Technology Conference, VTC Spring, IEEE, pp. 113-117, 2008.

[16] J. Anno, B. Leonard, D. Arjan, X. Fatos, and K. Akio, "Performance evaluation of two fuzzy-based cluster head selection systems for wireless sensor networks", Mobile Information Systems, Vol. 4, No. 4, pp. 297312,2008 .

[17] W. Pedrycz, and G. Fernando, "An introduction to fuzzy sets: analysis and design", Mit Press, 1998.

[18] B. E. Van, and D. B. Bernard, "Fast and accurate center of gravity defuzzification of fuzzy system outputs defined on trapezoidal fuzzy partitions", Fuzzy Sets and Systems, Vol. 157, No. 7,pp. 904-918,2006.

[19] J. S. Lee, and L. C. Wei, "Fuzzy-logic-based clustering approach for wireless sensor networks using energy predication", Sensors Journal, IEEE, Vol. 12, No. 9, pp. 2891-2897, 2012.

[20] M. H. Yin, and X. T. Li, "A hybrid bio-geography based optimization for permutation flow shop scheduling", Sci. Res. Essays, Vol. 6, No. 10, pp. 2078-2100, 2011.

[21] X. Li, W. Jinyan, Z. Junping, and Y. Minghao, "A perturb biogeography based optimization with mutation for global numerical optimization”, Applied Mathematics and Computation, Vol. 218, No. 2, pp. 598-609, 2011.

[22] X. Li, and Y. Minghao, "Hybrid differential evolution with biogeography-based optimization for design of a reconfigurable antenna array with discrete phase shifters", International Journal of Antennas and Propagation, 2011.

[23] G. Wang, G. Lihong, D. Hong, L. Luo, and W. Heqi, "Dynamic deployment of wireless sensor networks by biogeography based optimization algorithm", Journal of Sensor and Actuator Networks, Vol.1, No. 2, pp. 8696, 2012.

[24] L. A. Zadeh, "Soft computing and fuzzy approach", IEEE software, vol. 11, No. 6, pp. 48-56, Nov., 1994.

[25] R. V. Kulkarni, F. Anna, and K. V. Ganesh, "Computational intelligence in wireless sensor networks: A survey", Communications Surveys \& Tutorials, IEEE, Vol. 13, No. 1, pp. 68-96, Feb.,2011.

[26] K. Y. Cai, and Z. Lei, "Fuzzy reasoning as a control problem", Fuzzy Systems, IEEE Transactions, Vol. 16, No. 3, pp. 600-614,2008.

[27] D. Simon, "Biogeography-based optimization", IEEE Transactions, Vol. 12, No. 6, pp. 702-713, 2008.

[28] T. Runkler, "Selection of appropriate defuzzification methods using application specific properties", Fuzzy Systems, IEEE Transactions, Vol. 5, No. 1, pp. 72-79, 1997.

[29] W. R. Heinzelman, C. Anantha, and B. Hari, "Energy-efficient communication protocol for wireless microsensor networks", In System sciences, Proceedings of the 33rd annual Hawaii international conference, IEEE, pp. 10-pp. 2000.

[30] N. A. Latiff, C. T. Charalampos, and S. S. Bayan, "Performance comparison of optimization algorithms for clustering in wireless sensor networks", In Mobile Adhoc and Sensor Systems, MASS 2007. IEEE International Conference, pp. 1-4. IEEE, 2007.

[31] M. R. Minhas, G. Sathish, and L. Victor, "An online multipath routing algorithm for maximizing lifetime in wireless sensor networks", In Information Technology: New Generations, ITNG'09. Sixth International Conference, IEEE, pp. 581-586, 2009.

[32] M. A. Azim, and J. Abbas, "Performance evaluation of optimized forwarding strategy for flat sensor networks", In Global Telecommunications Conference, GLOBECOM'07. IEEE, pp. 710-714, 2007.

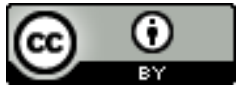

This work is licensed under a Creative Commons Attribution 4.0 International License.

DOI : 10.24297/ijmit.v12i2.6344 